Interactive comment on "Analysis of 24 years of mesopause region $\mathrm{OH}$ rotational temperature observations at Davis, Antarctica. Part 2:

Evidence of a quasi-quadrennial oscillation (QQO) in the polar mesosphere" by W. John R. French et al.

Anonymous Referee \#1

Received and published: 18 February 2020

Reviewer Report on the manuscript acp-2019-1097

Analysis of 24 years of mesopause region rotational temperature observations at Davis, Antarctica. Part 2: Evidence of quasi-quadrennial oscillation (QQO) in the polar mesosphere

By W.J.R.French, A.R.Klekociuk, and F.J.Mulligan 


\section{General Remarks}

1) The paper studies multi-annual variability in the lower and middle atmosphere up to the mesopause which is an interesting objective. 2) Emphasis is on quasi-quadrennial oscillations (QQO) observed in mesopause hydroxyl temperatures in a 24 year time series. 3) The variability signal is also seen in many other parameters as temperature, winds, geopotential, trace gas mixing ratios, SST, sea ice. These are obtained from various sources as satellites (MLS, SABER), ground based radar, ERA 5, etc.. 4) The analysis concerns vertical as well as meridional and zonal structures of the middle atmopsphere. This is very interesting and worth publishing! 5) However: More than half of the paper (pictures) deals with these parameters, only, and not with the Davis temperatures! The title of the paper is, therefore, inappropriate and misleading. It should be changed to something more general, and the manuscript should be rearranged accordingly. I know that this is not an easy task, as the interannual variability of the middle atmosphere is a very extended topic, and the data shown in the paper form only part of it. Nevertheles I recommend rewriting the paper in this direction, rather than turning it down. (To make it clear: I do not recommend that the authors write a review of middle atmosphere interannual variability, but that they state that their work forms an essential part of such a larger overview.) 6) The paper is well written, but many of the figures need improvement. 7) The paper is recommended for publication after major changes have been made.

\section{Major Comments}

1) Fig.1: a) The period of 4.2 years is not very convincing! In the years before 2006 the agreement of Davis-T, Saber-T, and the $4.2 \mathrm{yr}$ oscillation curve is marginal! Please give an error bar for the $4.2 \mathrm{yr}$ period value (see for instance Kalicinsky et al., ACP 16, 15033, 2016; Kalicinsky et al., JASTP 178, 7, 2018). b) How did you detrend solar cycle and long-term trend? Simultaneously or in an iteration?

Printer-friendly version

2) Fig. 1 and related text: Figure 10 might be moved to this part of the paper to illustrate 
that the interannual variability is fairly different in summer, winter, North and South.

3) Fig.2: a) This is mostly a global analysis, and only a small part is from $\mathrm{OH}$ temperatures. Hence, my General Remark \#5 applies. This is also the case in Fig.3 and many other places of the paper, especially for most of Section 4. My suggestions in the following assume a paper version in which title and text have been modified already. b) The "hashed areas" are indicated by crosses. These are difficult to discern! This also applies to the following figures, especially if the background colour is blue! The paper would become much more readable if this was improved! c) Fig.2b shows the vertical structure of the interannual variability, which is very interesting. However, the altitude resolution is poor: it only shows that the mesosphere differs from the stratosphere. As described in Section 2.2 there are more altitude levels available. Therefore please complement the left hand column of Fig. 2 by the altitude levels missing. This should show whether the vertical phase distribution is continuous or steplike (as the ones of Offermann et al., 2015).

4) Fig.3 and Fig.4: Please give time series as in the left hand panel in Fig.2.

5) Fig.5: Please give a time series for SST (near to Davis)!

6) Fig. 6: Please give time series!

7) Section 4.1: I understand that the authors are interested in showing a conection between SST and sea ice, and the upper atmosphere QQO. However, Sect.4.1 is not really suitable for this. The correlations discussed are marginal or non-existent (L387: $R=-0.09$ is not a correlation). The text refers to many literature papers that one would need to read in order to understand the text. Finally, correlation of a parameter below the tropopause with one above the tropopause is generally a delicate business, as is, for instance, indicated by Fig.3, 4. Altogether, a much mor extended analysis would be needed, as the authors state by themselves. As this is beyond the scope of this paper, I recommend to summarize this Section in a few sentences or omit it, at all. 
8) Section 4.3, L444pp: Obviously, the data of Dyrland and of Espy are Northern Hemisphere data. How does this compare to your $\mathrm{SH}$ results? Can you give a picture?

9) Section 4.4: $\mathrm{CO}$ is an important parameter, and its analysis is interesting. However, the correlation $R^{2}=0.13$ at 14 datapoints is barely significant.

10) L483: Do you mean concentrations or mixing ratios in the text and figures?

11) Fig.9a, L493pp: I could detect the "crosses of significance" only if I used a strong magnifying glas.

12) Sect.4.5; Lines 507, 510: If you omit two or four data points from a series of fourteen, the resulting conclusions are very dubious. Please phrase more cautiously!

13) Lines 529, 530: Apparently, WACCM does not detect your QQO, either! Why then show this Section 4.6 ?

14) $L$ 610-612: This is a misunderstanding: The periods cited are from the Duffin oscillator which is a non-linear oscillator in the ocean. However,the oscillations discussed by Offermann et al., 2015, are intrinsic in the atmosphere! These authors state that their results are not in contradiction to other authors who reported solar cycle harmonics. They note, however, that it is difficult to disentangle these two types (Section 6.2, last paragraph in that paper).

15) Summary, L 626pp: Please state clearly, that the Davis data are winter data, and that summer values are lacking. Fig.10 shows that there may be large differences!

Minor Comments

1) Line 38: relationship is suggesting

2) L 50: French et al., 2020

3) $L 80$ : including high

4) Fig.3 - 5: Please indicate location of Davis. 
5) Fig.8: Please give error bars.

6) Fig. 10: Please give error bars. Orange and red lines difficult to distinguish!

ACPD

Interactive comment on Atmos. Chem. Phys. Discuss., https://doi.org/10.5194/acp-2019-1097, 2020.

Interactive

comment 